\title{
On Exponential Stability of Composite Stochastic Control Systems
}

\author{
Fakhreddin Abedi and Wah June Leong \\ Department of Mathematics, Faculty of Science, Universiti Putra Malaysia, UPM, 43400 Serdang, \\ Selangor, Malaysia
}

Correspondence should be addressed to Fakhreddin Abedi; f_abedi1352@yahoo.com

Received 28 November 2012; Revised 29 January 2013; Accepted 31 January 2013

Academic Editor: Ryan Loxton

Copyright @ 2013 F. Abedi and W. J. Leong. This is an open access article distributed under the Creative Commons Attribution License, which permits unrestricted use, distribution, and reproduction in any medium, provided the original work is properly cited.

New results for exponential stability in probability of a composite stochastic control system are established. The main results of this paper enable us to derive sufficient conditions for exponential stability in $r$-th mean and almost sure exponential stability in probability of composite stochastic control system. Two numerical examples are given to illustrate the results.

\section{Introduction}

The aim of this paper is to study the exponential stability in probability in $r$-th mean (rESP) and almost sure exponential stability in probability (asESP) of a composite stochastic control system (CSCS)

$$
\begin{gathered}
d x=(f(x)+g(x, v) D v) d t+(h(x)+q(x, v) D v) d w_{t}, \\
d v=F(v, u) d t+G(v, u) d \beta_{t},
\end{gathered}
$$

where $x \in R^{n}, v \in R^{r}$, and $u$ is an $R^{p}$-valued measurable control law.

We consider the composite system (1) and introduce two subsystems

$$
\begin{aligned}
& d x=f(x) d t+h(x) d w_{t}, \\
& d v=F(v, u) d t+G(v, u) d \beta_{t},
\end{aligned}
$$

and we use the converse Lyapunov theorems established by Khasminiskii [1] and Spiliotis and Tsinias [2] to the concept of stochastic control Lyapunov function and extend the stabilization results proved by Spiliotis and Tsinias [2] for stochastic control systems (SCSs) to the larger class of CSCS (1) driven by two independent Wiener processes that have been considered in Florchinger [3], Boulanger [4], and Rusinek [5]. Sufficient conditions for rESP of stochastic systems (2) that guarantee rESP of CSCS (1) are derived, and we also establish sufficient conditions for asESP of CSCS (1).

In recent years, the stablizability of various types of stochastic systems has been studied for different concepts of stochastic stability (see, for instance, [1-17]). The global asymptotic stability in probability of stochastic systems by means of strict and output feedback laws has been considered by Krstic and Deng [6] and Deng and Krstic [8], where backstepping design procedure is proposed for stochastic systems. Tsinias [7] extended the input-to-state stability results of Sontag and Wang [18] in deterministic case and obtained exponential input-to-state stability in probability for stochastic system and derived sufficient conditions for global stabilization of this system by means of static and dynamic output feedback. Caraballo and Liu [9] developed some criteria for the almost sure and mean square exponential stability in probability of nonlinear stochastic equations of monotone type. Spiliotis and Tsinias [2] derived control Lyapunov function and established rESP and asESP of stochastic differential systems. The asymptotic and exponential stability of stochastic differential systems (SDSs) has been studied by Mao [10], Liu and Raffool [11], Lan and Dang [12], Luo [13], Abedi et al. [14], Abedi and Leong [15], Khasminiskii [1], and Kushner [16]. Michel [17] established asymptotic and exponential stability in probability for some classes 
of continuous and discrete-parameter stochastic composite systems. Later, Florchinger [3] and Boulanger [4] developed sufficient conditions for asymptotic stability in probability and exponential stability in mean square for a special case of our CSCS (1). The sufficient conditions for exponential stability in probability of nonlinear stochastic systems and special case of our CSCS (1) have been derived by Rusinek [5].

The structure of the paper is as follows. In Section 2, we introduce a class of CSCS and we also recall some basic definitions and results concerning rESP and asESP property. In Section 3, we state and prove the main results of the paper. Finally in Section 4, we give two numerical examples illustrating our results.

\section{Fundamental Definitions and Results}

In this section, we introduce the class of stochastic systems and recall some definitions and results concerning exponential stability in probability of these systems. For a complete presentation of exponential stability, we refer the reader to the book of Khasminiskii [1] and the paper of Spiliotis and Tsinias [2].

Let $(\Omega, F, P)$ be a complete probability space and denote by $\left(w_{t}\right)_{t \geq 0}$ a standard $R^{m}$-valued Wiener process defined on this space.

We consider the SDS

$$
d x=f(x) d t+h(x) d w_{t}
$$

where the following conditions hold:

(i) $x$ is given in $R^{n}$,

(ii) $f: R^{n} \rightarrow R^{n}$ and $h: R^{n} \rightarrow R^{n \times m}$ are Lipschitz functionals in $R^{n}$ with $f(0)=0, h(0)=0$, and there exists a constant $C \geq 0$ such that the following growth condition holds:

$$
|f(x)-f(y)|+|h(x)-h(y)| \leq C|x-y|
$$

for any $x \in R^{n}$.

Under restriction on growth (4), for any $x \in R^{n}$, that guarantees existence and uniqueness of solution $x(t)=$ $x\left(t, t_{0}, x_{0}\right)$ for (3) starting from $x_{0}$ at time $t_{0}$, we recall the following definition of exponential stability in mean square and a converse Lyapunov theorem given by Khasminskii [1] as follows.

Definition 1. The origin of the SDS (3) is exponentially stable in probability in mean square if, and only if, there exist constants $c_{1}, c_{2}>0$ such that

$$
E\left(\left|x_{t}\left(t, t_{0}, x\right)\right|^{2}\right) \leq c_{1}|x|^{2} e^{-c_{2}\left(t-t_{0}\right)},
$$

for any $x \in R^{n}$, and $t \geq t_{0}$.

Theorem 2. Suppose that the origin $x_{t}=0$ of the SDS (3) is exponentially stable in probability in mean square. Then, there exists a Lyapunov function $\Phi$ defined on $R^{n}\left(\Phi \in C^{2}\left(R^{n}, R^{+}\right)\right)$ and positive constants $a_{i}, 1 \leq i \leq 5$, such that

$$
\begin{gathered}
a_{1}|x|^{2} \leq \Phi(x) \leq a_{2}|x|^{2}, \\
|\nabla \Phi(x)| \leq a_{3}|x|, \quad\left|\nabla^{2} \Phi(x)\right| \leq a_{4}, \\
\operatorname{D} \Phi(x)=\sum_{i=1}^{n} f^{i}(x) \frac{\partial \Phi(x)}{\partial x_{i}}+\frac{1}{2} \sum_{i, j=1}^{n} \sum_{k=1}^{m} h_{k}^{i} h_{k}^{j} \frac{\partial^{2} \Phi(x)}{\partial x_{i} \partial x_{j}} \\
\leq-a_{5}|x|^{2},
\end{gathered}
$$

where $\mathbf{D}$ is the infinitesimal generator for the stochastic process solution of the SDS (3).

Let us denote by $\left(\beta_{t}\right)_{t \geq 0}$ a standard $R^{q}$-valued Wiener process defined on $(\Omega, \mathrm{F}, P)$.

Now, we consider the SCS

$$
d v=F(v, u) d t+G(v, u) d \beta_{t},
$$

where

(1) $u$ is an $R^{p}$-valued measurable control law,

(2) $F$ and $G$ are Lipschitz functionals mapping of $R^{r} \times R^{p}$ into $R^{r}$ and $R^{r} \times R^{q}$, respectively, such that $F(0,0)=$ $G(0,0)=0$, and there exists constant $C \geq 0$ such that for any $v \in R^{r}$ and $u \in R^{p}$ the following growth condition holds:

$$
|F(v, u)|+|G(v, u)| \leq C(1+|v|+|u|) .
$$

Under restriction on growth (8) (see for instance Arnold [19]), for every input $u \in R^{p}, v_{0} \in R^{r}$, and $t_{0} \in R$, there exists a unique solution $V\left(t, t_{0}, v_{0}, u\right)$ of (7) starting from $v_{0}$ at time $t=t_{0}$ which is defined for all $t \geq t_{0}$ and almost all $\beta \in \Omega$. For simplicity let $V(t)=V\left(t, t_{0}, v, u\right)$, and we recall the following definition of rESP, asESP, and a converse Lyapunov theorem established by Spiliotis and Tsinias [2] as follows.

Definition 3. The origin of the SCS (7) is rESP for some $r>0$ if, and only if, there exist constants $c_{1}, c_{2}>0$ such that

$$
E\left(|V(t)|^{r}\right) \leq c_{1}|v|^{r} e^{-c_{2}\left(t-t_{0}\right)},
$$

for any $v \in R^{r}$, and $t \geq t_{0}$.

Definition 4. The SCS (7) is said to satisfy the exponential Lyapunov condition if there exists a Lyapunov function $\Phi$ : $R^{r} \rightarrow R^{+}$of class $C^{2}\left(R^{r} \backslash\{0\}\right)$ and positive constants $a_{i}, 1 \leq$ $i \leq 5$, such that

$$
\begin{gathered}
a_{1}|v|^{r} \leq \Phi(v) \leq a_{2}|v|^{r} \\
|\nabla \Phi(v)| \leq a_{3}|v|^{r-1}, \quad v \neq 0 \\
\left|\nabla^{2} \Phi(v)\right| \leq a_{4}|v|^{r-2}, \quad v \neq 0 \\
\mathbf{Y} \Phi(v)=\sum_{i=1}^{n} F(v, u) \frac{\partial \Phi(v)}{\partial v_{i}}+\frac{1}{2} \sum_{i, j=1}^{n} G(v, u) G(v, u)^{T} \frac{\partial^{2} \Phi(v)}{\partial v_{i} v_{j}} \\
\leq-a_{5}|v|^{r}
\end{gathered}
$$


where $\mathbf{Y}$ is the infinitesimal generator for the stochastic process solution of the SCS (7).

Theorem 5. Suppose that the origin of the SCS (7) is rESP. Then, there exists a Lyapunov function $\Phi: R^{r} \rightarrow R^{+}$of class $C^{2}\left(R^{r} \backslash\{0\}\right)$ which satisfies all conditions (10)-(13).

Note that Definition 3 and Theorem 5 provided in Spiliotis and Tsinias [2] are an extension of Definition 1 and Theorem 2, respectively, established in Khasminskii [1].

Definition 6. The origin of the SCS (7) is asESP for some $r>0$ if, and only if, there exists a constant $c>0$ and a random variable $0 \leq B_{v, u}<\infty$, such that

$$
|V(t)| \leq B_{v, u} e^{-c\left(t-t_{0}\right)},
$$

for any $v \in R^{r}, t \geq t_{0}$, and for almost all $\beta$.

We now derive the Florchinger's decomposition [20] of the functions

$$
\begin{gathered}
F(v, y)=f(v, l(v, y)) h(v, y), \\
G(v, y)=g(v, y) \sqrt{h(v, y),}
\end{gathered}
$$

and we consider the SCS (7) in the form

$$
\begin{aligned}
d\left(\begin{array}{l}
v \\
y
\end{array}\right)= & \left(\begin{array}{c}
f(v, l(v, y)) h(v, y) \\
u
\end{array}\right) d t \\
& +\left(\begin{array}{c}
g(v, y) \sqrt{h(v, y)} \\
0
\end{array}\right) d \beta_{t},
\end{aligned}
$$

where $l$ is a function mapping $R^{r} \times R^{p}$ into $R^{p}$ and $h$ is a nonnegative function mapping $R^{r} \times R^{p}$ into $R$. In the following theorem, we use an explicit formula of a feedback law established in $[15,20]$ and exhibit the rESP property for the resulting closed-loop system deduced from (7) or, equivalently, the resulting closed-loop system deduced from (16).

Theorem 7. Let $V(t)=V\left(t, t_{0}, v, y\right)$. Suppose that the SCS (7) or, equivalently, the SCS (16) satisfies the exponential Lyapunov condition, and there exists a $C^{\infty}$ function $\Gamma: R^{r} \times R^{p} \rightarrow R^{p}$ such that for any $(v, y) \in R^{r} \times R^{p}$,

$$
\begin{aligned}
\gamma(v, y)= & \left\langle f(v, \varphi(v)), \frac{\partial \phi}{\partial v}(v, y)\right\rangle h(v, y) \\
& +\frac{1}{2} h(v, y) \sum_{i, j=1}^{m}\left(g(v, y) g(v, y)^{T}\right)_{i, j} \frac{\partial^{2} \phi(v, y)}{\partial v_{i} \partial v_{j}} \\
& +\left\langle\Gamma(v, y), \frac{\partial \phi}{\partial y}(v, y)\right\rangle \leq 0 .
\end{aligned}
$$

Then, the feedback law

$$
\begin{aligned}
k_{1}(v, y)= & -M(v, y)^{T} N(v, y)^{T} \frac{\partial W(V(t))}{\partial v} \\
& +\Gamma(v, y)-\frac{\partial \phi}{\partial y}(v, y),
\end{aligned}
$$

where $M(v, y)(\partial \phi / \partial y)(v, y)=(l(v, y)-\varphi(v)) h(v, y)$, $N(v, y)=\int_{0}^{1}(\partial F / \partial u)(v, t l(v, y)+(1-t) \varphi(v)) d t, \phi: R^{r} \times$ $R^{p} \rightarrow R$ is a smooth and nonnegative function, $W(V(t))=$ $\Phi(v)+\phi(v, y)$, and $\varphi(v): R^{r} \rightarrow R^{p}$ is a smooth function that guarantees the resulting closed-loop system deduced from (7) or, equivalently, that guarantees the resulting closed-loop system deduced from (16) is rESP.

Proof. Suppose that there exists a positive function $W(V(t))$ such that the SCS (7) or, equivalently, the SCS (16) satisfies the exponential Lyapunov condition. Then from (13) we have

$$
\begin{aligned}
\frac{d}{d t} E W(V(t)) & =E\left(\mathbf{Y}_{1} W(V(t))\right) \\
& =E\left[\mathbf{D}_{0} \Phi(v) h(v, y)+\gamma(v, y)\right. \\
& \left.\quad-\left\|\frac{\partial \phi}{\partial y}(v, y)\right\|^{2}\right] \\
& \leq-a_{5} E\left(|V(t)|^{r}\right),
\end{aligned}
$$

where $\mathbf{D}_{0}$ is the infinitesimal generator of the resulting closed-loop system deduced from (7) and $\mathbf{Y}_{1}$ is the infinitesimal generator for the stochastic process solution of the resulting closed-loop system deduced from (16) as follows:

$$
\begin{aligned}
\mathbf{Y}_{1} W(V(t)) \\
=(\langle f(v, \varphi(v)), \nabla \Phi(v)\rangle k \\
\left.\quad+\frac{1}{2} \sum_{i, j=1}^{m}\left(g(v, y) g(v, y)^{T}\right)_{i, j} \frac{\partial^{2} \Phi(v)}{\partial v_{i} \partial x_{j}}\right) \\
\quad \times h(v, y)+\left\langle f(v, \varphi(v)), \frac{\partial \phi}{\partial v}(v, y)\right\rangle h(v, y) \\
+\frac{1}{2} h(v, y) \sum_{i, j=1}^{m}\left(g(v, y) g(v, y)^{T}\right)_{i, j} \frac{\partial^{2} \phi(v, y)}{\partial v_{i} \partial v_{j}} \\
+\left\langle\Gamma(v, y), \frac{\partial \phi}{\partial y}(v, y)\right\rangle-\left\|\frac{\partial \phi}{\partial y}(v, y)\right\|^{2} .
\end{aligned}
$$

The desired condition (9) is a direct consequence of inequality (10) and (19). Therefore, the resulting closed-loop system deduced from SCS (16) satisfies in rESP property at the origin.

We will now turn the attention to a general composite stochastic system and provide some results related to the rESP of this system.

Let $\left\{\beta_{t}, t \in R^{+}\right\}$be a standard $R^{q}$-valued Wiener process defined on the space $(\Omega, \mathrm{F}, P)$ independent of the Wiener process $\left\{w_{t}, t \in R^{+}\right\}$. 
Consider the pair of stochastic processes solution $\left(x_{t}, v_{t}\right) \in R^{n} \times R^{r}$ of the CSCS

$$
\begin{gathered}
d x=(f(x)+g(x, v) D v) d t+(h(x)+q(x, v) D v) d w_{t} \\
d v=F(v, u) d t+G(v, u) d \beta_{t}
\end{gathered}
$$

where

(1) $x \in R^{n}, v \in R^{r}$, and $D$ is a matrix function with value in $M_{r \times r}(R)$,

(2) $f$ and $h$ are functionals in $C^{2}\left(R^{n}, R^{n}\right)$ and $C^{2}\left(R^{n}, R^{n \times m}\right)$, respectively, such that $f(0)=0$ and $h(0)=0$,

(3) $g: R^{n} \times R^{r} \rightarrow R^{n \times r}$ and $q: R^{n} \times R^{r} \rightarrow R^{n \times m}$ are Lipschitz functionals mapping such that there exists a nondecreasing scalar function $\alpha(|v|) \geq 0$ bounded for all $v$ such that

$$
\begin{array}{r}
|g(x, v)|+|q(x, v)| \leq \alpha(|v|)|x|, \\
\forall(x, v) \in R^{n} \times R^{r},
\end{array}
$$

(4) $u$ is an $R^{p}$-valued measurable control law,

(5) $F: R^{r} \times R^{p} \rightarrow R^{r}$ and $G: R^{r} \times R^{p} \rightarrow R^{r \times q}$ are Lipschitz functionals mapping vanishing at the origin, and there exists a constant $C \geq 0$ such that the following growth condition holds:

$$
|F(v, u)|+|G(v, u)| \leq C(1+|v|+|u|),
$$

for any $(v, u) \in R^{r} \times R^{p}$.

Definition 8. We say that an input measurable function $u$ : $R^{n} \times R^{r} \rightarrow R^{p}, u(0,0)=0$ is a stabilizing feedback law for the CSCS (21) if, and only if, the origin of the resulting closedloop system

$$
\begin{gathered}
d x=(f(x)+g(x, v) D v) d t+(h(x)+q(x, v) D v) d w_{t} \\
d v=F(v, u(x, v)) d t+G(v, u(x, v)) d \beta_{t}
\end{gathered}
$$

is $\mathrm{rESP}$.

Suppose that there exists functionals $F_{1}: R^{r} \rightarrow R^{r}, F_{2}$ : $R^{r} \rightarrow R^{r \times p}, G_{1}: R^{r} \rightarrow R^{r \times q}$, and $G_{2}: R^{r} \rightarrow R^{r \times p \times q}$ such that

$$
F(v, u)=F_{1}(v)+F_{2}(v) u, \quad G(v, u)=G_{1}(v)+G_{2}(v) u
$$

$$
d v=\left(F_{1}(v)+F_{2}(v) u\right) d t+\left(G_{1}(v)+G_{2}(v) u\right) d \beta_{t},
$$

for any $(v, u) \in R^{r} \times R^{p}$. As mentioned above, we can consider the general SCS (7) into the form of (16). This form of (16) is the same as (26), which is a special case of (7). By taking into account (7), (16), and (26), the CSCS (21) is rewritten as

$$
\begin{gathered}
d x=(f(x)+g(x, v) D v) d t+(h(x)+q(x, v) D v) d w_{t}, \\
d v=\left(F_{1}(v)+F_{2}(v) u\right) d t+\left(G_{1}(v)+G_{2}(v) u\right) d \beta_{t}
\end{gathered}
$$

In the next section, we will establish a state feedback law that guarantees the satisfaction of rESP and asESP property for CSCS (27).

\section{Exponential Stability of Composite Systems}

Our aims of this section are twofold. On one hand, we study the problem of finding state feedback law that guarantees that the CSCS (27) satisfies rESP property. We derive sufficient conditions for rESP of this system. On the other hand, we establish sufficient conditions for asESP of the CSCS (27).

In the following theorem, we suppose that the function $g$ and $q$ are bounded on $R^{n} \times R^{p}$ and $U$ is the set of admissible control and establish a sufficient conditions for rESP property of CSCS (27). Theorem 9 is a stochastic extension of Proposition 3.1, Theorem 5.1, and Theorem 4.1 stated in Spiliotis and Tsinias [2], Florchinger [3], and Boulanger [4], respectively, to a general composite stochastic system. Both the results and the proof used in this theorem, however, are different from those in [2-4]. Furthermore, we can consider the exponential stability in mean square results of Florchinger [3] and Boulanger [4] as a special case of our rESP results (Theorem 9) where $r=2$.

Theorem 9. Let $V(t)=V\left(t, t_{0}, x, v\right)$. Suppose that the SDS

$$
d x=f(x) d t+h(x) d w_{t}
$$

satisfies rESP property, and there exists a state control law $k_{1}$ : $R^{n} \times R^{r} \rightarrow R^{p}$,

$$
\begin{aligned}
k_{1}(x, v)= & -M(x, v)^{T} N(x, v)^{T} \frac{\partial W(V(t))}{\partial x} \\
& +\Gamma(x, v)-\frac{\partial \phi}{\partial v}(x, v),
\end{aligned}
$$

where $W(V(t))=\Phi_{2}(x)+\phi(x, v)$, that guarantees that the resulting closed-loop system

$$
\begin{aligned}
d v= & \left(F_{1}(v)+F_{2}(v) k_{1}(x, v)\right) d t \\
& +\left(G_{1}(v)+G_{2}(v) k_{1}(x, v)\right) d \beta_{t}
\end{aligned}
$$

satisfies the rESP property. Furthermore, assume that

$$
D v=F_{2}(v)^{T} \nabla \Phi_{2}(v)+\nabla \Phi_{2}(v) G_{2}(v)^{T} .
$$

Then, the state control law

$$
k(x, v)=k_{1}(x, v)-g(x, v)^{T} \nabla \Phi_{1}(x)-q(x, v)^{T} \nabla \Phi_{1}(x),
$$

where $\Phi_{1}$ is a smooth Lyapunov function corresponding to the SCS (28), guarantees that the CSCS (27) satisfies rESP.

Proof. Suppose that the origin of the stochastic system (28) satisfies the rESP property. Then by the converse Lyapunov Theorem 5, there exists a Lyapunov function $\Phi_{1}(x)$ and positive constants $a_{i}, 1 \leq i \leq 5$, such that (10)-(12) hold and

$$
\begin{aligned}
\mathbf{D} \Phi_{1}(x) & =\sum_{i=1}^{n} f^{i}(x) \frac{\partial \Phi_{1}(x)}{\partial x_{i}}+\frac{1}{2} \sum_{i, j=1}^{n} \sum_{k=1}^{m} h_{k}^{i} h_{k}^{j} \frac{\partial^{2} \Phi_{1}(x)}{\partial x_{i} \partial x_{j}} \\
& \leq-a_{5}|x|^{r} .
\end{aligned}
$$


On the other hand, since the origin is a rESP for the closedloop system (30), then by converse Lyapunov Theorem 5, there exists a Lyapunov function $\Phi_{2}(v)$ and positive constants $a_{i}^{\prime}, 1 \leq i \leq 5$, such that the following conditions hold:

$$
\begin{gathered}
a_{1}^{\prime}|v|^{r} \leq \Phi_{2}(v) \leq a_{2}^{\prime}|v|^{r}, \\
\left|\nabla \Phi_{2}(v)\right| \leq a_{3}^{\prime}|v|^{r-1}, \quad v \neq 0, \\
\left|\nabla^{2} \Phi_{2}(v)\right| \leq a_{4}^{\prime}|v|^{r-2}, \quad v \neq 0, \\
\mathbf{Y}_{2} \Phi_{2}(v, u)=\sum_{i=1}^{n} F(v, u) \frac{\partial \Phi(v)}{\partial v_{i}} \\
+\frac{1}{2} \sum_{i, j=1}^{n} G(v, u) G(v, u)^{T} \frac{\partial^{2} \Phi(v)}{\partial v_{i} v_{j}} \\
\leq-a_{5}^{\prime}|v|^{r},
\end{gathered}
$$

where $\mathbf{Y}_{2}$ is the infinitesimal generator for the stochastic process solution of the resulting closed-loop system (30). Consider the composite function

$$
\Phi(V(t))=\Phi_{1}(x)+\Phi_{2}(v)
$$

for any $(x, v) \in R^{n} \times R^{r}$. Obviously, $\Phi(V(t))$ is a Lyapunov function. We will show that $\Phi(V(t))$ satisfies in the exponential Lyapunov condition. A simple calculation shows that conditions (10)-(12) are satisfied. It remains to establish condition (13). Substituting $k(x, v)$ into the closedloop system deduced from CSCS (27), we have

$$
\begin{aligned}
d x=( & f(x)+g(x, v) D v) d t+(h(x)+q(x, v) D v) d w_{t} \\
d v=( & F_{1}(v)+F_{2}(v) k_{1}(v) \\
& -F_{2}(v) g(x, v)^{T} \nabla \Phi_{1}(x) \\
& \left.-F_{2}(v) q(x, v)^{T} \nabla \Phi_{1}(x)\right) d t \\
+ & \left(G_{1}(v)+G_{2}(v) k_{1}(v)\right. \\
& \quad-G_{2}(v) g(x, v)^{T} \nabla \Phi_{1}(x) \\
& \left.-G_{2}(v) q(x, v)^{T} \nabla \Phi_{1}(x)\right) d \beta_{t} .
\end{aligned}
$$

Denoting $\mathbf{D}_{v}$ as the infinitesimal generator of the stochastic process solution of the resulting closed-loop system (39) yields

$$
\begin{aligned}
& \mathbf{D}_{v} \Phi(V(t)) \\
&= \mathbf{D} \Phi_{1}(x)+\nabla \Phi_{1}(x)^{T} g(x, v) D v \\
&+\nabla \Phi_{1}(x)^{T} q(x, v) D v+\mathbf{Y}_{2} \Phi_{2}(v) \\
&-\nabla \Phi_{1}(v)^{T} F_{2}(v) g(x, v)^{T} \nabla \Phi_{1}(x) \\
&-\nabla \Phi_{2}(v)^{T} F_{2}(v) q(x, v)^{T} \nabla \Phi_{1}(x) \\
&-\nabla \Phi_{2}(v)^{T} G_{2}(v) g(x, v)^{T} \nabla \Phi_{1}(x) \\
&-\nabla \Phi_{2}(v)^{T} G_{2}(v) q(x, v)^{T} \nabla \Phi_{1}(x),
\end{aligned}
$$

for any $(x, v) \in R^{n} \times R^{r}$. Substituting (31) into (40), we get

$$
\mathbf{D}_{v} \Phi(V(t))=\mathbf{D} \Phi_{1}(x)+\mathbf{Y}_{2} \Phi_{2}(v) .
$$

Thus, we obtain by taking into account (33) and (37) that

$$
\begin{aligned}
\mathbf{D}_{v} \Phi(V(t)) & =\mathbf{D} \Phi_{1}(x)+\mathbf{Y}_{2} \Phi_{2}(v) \\
& \leq-a_{5}|x|^{r}-a_{5}^{\prime}|v|^{r}
\end{aligned}
$$

From (42) and the above results, we have the Laypunov function $\Phi(V(t))$ satisfies in exponential Lyapunov condition. Then, according to the Lyapounov theorem established in Spiliotis and Tsinias [2, Proposition 3.2], the origin of the CSCS (27) satisfies rESP property.

In the following theorem, we show that existence of a Lyapunov function satisfying the exponential Lyapunov condition implies rESP and asESP of the CSCS (27).

Theorem 10. Assume that there exists a $C^{2}\left(R^{r} \backslash\{0\}\right)$ function $\Phi$ satisfying the exponential Lyapunov condition. Then,

(i) the origin of the CSCS (27) is rESP,

(ii) the origin of the CSCS (27) is asESP.

Proof. Part (i) Assume that there exists a positive function $\Phi(V(t))$ such that the CSCS (27) or equivalently the CSCS (39) satisfies the exponential Lyapunov condition. Then from (13) we have

$$
\begin{aligned}
\frac{d}{d t} E \Phi(V(t)) & =E\left(\mathbf{D}_{v} \Phi(V(t))\right) \\
& =E\left(\mathbf{D} \Phi_{1}(x)+\mathbf{Y}_{2} \Phi_{2}(v)\right) \\
& \leq-a_{5} E\left(|x|^{r}\right)-a_{5}^{\prime} E\left(|v|^{r}\right),
\end{aligned}
$$

where $\Phi_{1}$ and $\Phi_{2}$ are Lyapunov functions corresponding to the SDS (28) and SCS (30), respectively, and $D_{v}$ is the infinitesimal generator of the stochastic process solution of the resulting closed-loop system (39). The desired condition (9) is a direct consequence of inequality (10) and (43). Therefore, CSCS (27) satisfies in rESP property at the origin. 
Part (ii) Suppose that there exists a positive composite function

$$
\Phi(V(t))=\Phi_{1}(x) \exp ^{\left(\left(a_{5} / a_{2}\right) t\right)}+\Phi_{2}(v) \exp ^{\left(\left(a_{5}^{\prime} / a_{2}^{\prime}\right) t\right)},
$$

where $\Phi_{1}$ and $\Phi_{2}$ are Lyapunov functions corresponding to the SDS (28) and SCS (30), respectively, such that the CSCS (27) satisfies the exponential Lyapunov condition. Then, by taking into account (10)-(13), (40), (31), and (44), we obtain

$$
\begin{aligned}
\mathbf{D}_{v} \Phi(V(t)) & =\mathbf{D} \Phi_{1}(x) \exp { }^{\left(\left(a_{5} / a_{2}\right) t\right)}+\mathbf{Y}_{2} \Phi_{2}(v) \exp \left(\left(a_{5}^{\prime} / a_{2}^{\prime}\right) t\right) \\
& \leq 0
\end{aligned}
$$

From (44) and (45) and $\Phi(V(t)) \geq 0$, we have $\Phi_{t}=\Phi(V(t))$ is a supermartingale. Therefore, for some random $B_{x, v}$, it yields

$$
\lim _{t \rightarrow \infty} \Phi(V(t)) \leq B_{x, v}<\infty . \quad \text { a.s. }
$$

From (46), we have $\Phi(V(t)) \leq K_{x, v}<\infty$ for all $t \geq 0$, almost surely, for some random $K_{x, v} \geq B_{x, v}$. The latter in conjunction with (10), (43), and (44) implies (14). Thus, CSCS (27) satisfies in asESP property at the origin.

Remark 11. Comparing the existing results in [2-8] we can make the following summaries.

(i) Theorem 9 is a stochastic extension of Proposition 3.1, Theorem 5.1, and Theorem 4.1 stated in Spiliotis and Tsinias [2], Florchinger [3], and Boulanger [4], respectively, to a general composite stochastic system. Both the results and the proof used in this theorem, however, are different from those in [2-4]. Furthermore, we can consider the exponential stability in mean square results of Florchinger [3] and Boulanger [4] as a special case of our rESP results (Theorem 9) where $r=2$.

(ii) The rESP and asESP results for stochastic system proved in Spiliotis and Tsinias [2] are a special case of our rESP and asESP results for CSCS (21). In addition, the existing exponential stability results established in [2-8] do not permit us to make a conclusion about rESP and asESP for the broader class of CSCS (21) at the origin, whereas the results of this paper are still valid.

\section{Applications}

This section illustrates applicability of our results by designing the following two numerical examples.

Example 12. Consider the SDS

$$
d x=-2 x d t+x d w_{t}
$$

where $\left(w_{t}\right)_{t \geq 0}$ is a standard real-valued Wiener process and $x \in R$. Assume that $\Phi(x)=x^{2}$ is a smooth Lyapunov function corresponding to the SDS (47). Then, a simple calculation shows that the SDS (47) satisfies rESP.
Next, consider the SCS

$$
d v=2 v^{2} u d t+\frac{1}{2} v u d \beta_{t}
$$

where $\left(\beta_{t}\right)_{t \geq 0}$ is a standard real-valued Wiener process, $v \in R$, and $u$ is a real-valued measurable control law. Obviously,

$$
\begin{aligned}
k_{1}(x, v)= & -M(x, v)^{T} N(x, v)^{T} \frac{\partial W(V(t))}{\partial x} \\
& +\Gamma(x, v)-\frac{\partial \phi}{\partial v}(x, v) \\
= & -10 x^{3}-4 x^{2} v-v,
\end{aligned}
$$

where $\phi(x, v)=(1 / 2)(2 v+x)^{2}, l(x, v)=v, h(x, v)=1, \varphi(x)=$ $-2 x, \Gamma(x, v)=2 x, M(x, v)=1, N(x, v)=2 x^{2}$, and

$$
W(V(t))= \begin{cases}\frac{1}{2}\left[v^{2}+(x+2 v)^{2}\right], & (x, v) \neq 0, \\ 0, & (x, v)=0,\end{cases}
$$

renders the resulting closed-loop system

$$
d v=2 v^{2} k_{1}(x, v) d t+\frac{1}{2} v k_{1}(x, v) d \beta_{t}
$$

satisfies the rESP property. Thus, according to Theorem 9, there exists the state control law

$$
\begin{aligned}
k(x, v) & =k_{1}(x, v)-g(x, v)^{T} \nabla \Phi(x)-q(x, v)^{T} \nabla \Phi(x) \\
& =-10 x^{3}-4 x^{2} v-v-4 x
\end{aligned}
$$

that guarantees the CSCS

$$
\begin{gathered}
d x=(-2 x+v) d t+(x+v) d w_{t}, \\
d v=2 v^{2} u d t+\frac{1}{2} v u d \beta_{t},
\end{gathered}
$$

where $g(x, v)=q(x, v)=1$ and $D=I$ is the identity matrix in $M_{r \times r}(R)$ satisfies rESP property.

Example 13. Consider the CSCS

$$
\begin{gathered}
d x=(-2 x+v) d t+(x+v) d w_{t}, \\
d v=v d t+(5 v-5 v u) d \beta_{t},
\end{gathered}
$$

where $x, v \in R$ and $u \in[-(1 / 2),(1 / 2)]$. Consider the Lyapunov function candidate $\Phi(V(t))=|x|^{r}+|v|^{r}, 0<$ $r<1 / 5$. A simple calculation shows that $\Phi(V(t))$ satisfies the exponential Lyapunov condition. Then, according to Theorem 10, the origin is rESP and asESP.

\section{Conclusions}

In this paper, we have provided the new results for rESP and asESP of the CSCS

$$
\begin{gathered}
d x=(f(x)+g(x, v) v) d t+(h(x)+q(x, v) v) d w_{t}, \\
d v=F(v, u) d t+G(v, u) d \beta_{t} .
\end{gathered}
$$


We have used the converse Lyapunov theorems established by Khasminiskii [1] and Spiliotis and Tsinias [2] to the concept of stochastic control Lyapunov function and extended the stabilization results proved by Spiliotis and Tsinias [2] for SCS to the larger class of CSCS (55) driven by two independent Wiener processes that have been considered in Florchinger [3], Boulanger [4], and Rusinek [5]. We have also derived sufficient conditions for rESP and asESP of CSCS (55).

\section{References}

[1] R. Z. Khasminskii, Stochastic Stability of Differential Equations, vol. 7, Sijthoff \& Noordhoff, Alphen aan den Rijn, The Netherlands, 1980

[2] J. Spiliotis and J. Tsinias, "Notions of exponential robust stochastic stability, ISS and their Lyapunov characterization," International Journal of Robust and Nonlinear Control, vol. 13, no. 2, pp. 173-187, 2003.

[3] P. Florchinger, "Global stabilization of composite stochastic systems," Computers \& Mathematics with Applications, vol. 33, no. 6, pp. 127-135, 1997.

[4] C. Boulanger, "Stabilization of a class of nonlinear composite stochastic systems," Stochastic Analysis and Applications, vol. 18, no. 5, pp. 723-735, 2000.

[5] R. L. Rusinek, "String stability of singularly perturbed stochastic systems," Annales Mathematicae Silesianae, vol. 16, pp. 43-55, 2003.

[6] M. Krstic and H. Deng, Stabilization of Uncertain Nonlinear Systems, Springer, New York, NY, USA, 1998.

[7] J. Tsinias, "The concept of "exponential input to state stability" for stochastic systems and applications to feedback stabilization," Systems \& Control Letters, vol. 36, no. 3, pp. 221-229, 1999.

[8] H. Deng and M. Krstić, "Output-feedback stochastic nonlinear stabilization," IEEE Transactions on Automatic Control, vol. 44, no. 2, pp. 328-333, 1999.

[9] T. Caraballo and K. Liu, "On exponential stability criteria of stochastic partial differential equations," Stochastic Processes and Their Applications, vol. 83, no. 2, pp. 289-301, 1999.

[10] X. Mao, Exponential Stability of Stochastic Differential Equations, vol. 182, Marcel Dekker, New York, NY, USA, 1994.

[11] R. Liu and Y. Raffoul, "Boundedness and exponential stability of highly nonlinear stochastic differential equations," Electronic Journal of Differential Equations, vol. 143, pp. 1-10, 2009.

[12] T. T. T. Lan and N. H. Dang, "Exponential stability of nontrivial solutions of stochastic differential equations," Scientia A, vol. 21, pp. 97-106, 2011.

[13] J. Luo, "Exponential stability for stochastic neutral partial functional differential equations," Journal of Mathematical Analysis and Applications, vol. 355, no. 1, pp. 414-425, 2009.

[14] F. Abedi, M. A. Hassan, and N. M. Arifin, "Lyapunov function for nonuniform in time global asymptotic stability in probability with application to feedback stabilization," Acta Applicandae Mathematicae, vol. 116, no. 1, pp. 107-117, 2011.

[15] F. Abedi and W. J. Leong, "Dynamic robust stabilization of stochastic differential control systems," IMA Journal of Mathematical Control and Information, 2013.

[16] H. J. Kushner, "On the stability of processes defined by stochastic difference-differential equations," Journal of Differential Equations, vol. 4, pp. 424-443, 1968.
[17] A. N. Michel, "Stability analysis of stochastic composite systems," IEEE Transactions on Automatic Control, vol. AC-20, pp. 246-250, 1975.

[18] D. E. Sontag and Y. Wang, "On characterizations of input-tostate stability properties," Systems and Control Letters, vol. 24, pp. 226-231, 1995.

[19] L. Arnold, Stochastic Differential Equations: Theory and Applications, John Wiley \& Sons, New York, NY, USA, 1974.

[20] P. Florchinger, "A new decomposition method for stochastic dynamic stabilization," Computers \& Mathematics with Applications, vol. 29, no. 3, pp. 31-36, 1995. 


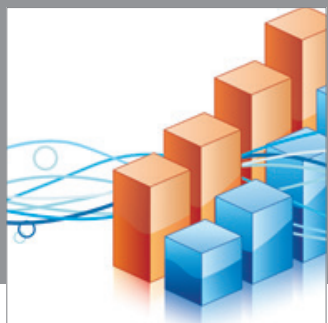

Advances in

Operations Research

mansans

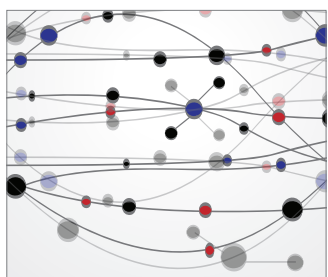

The Scientific World Journal
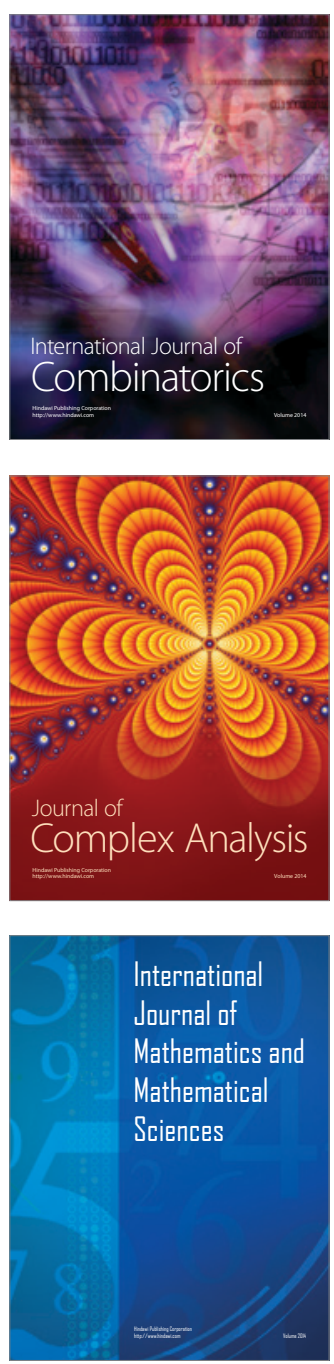
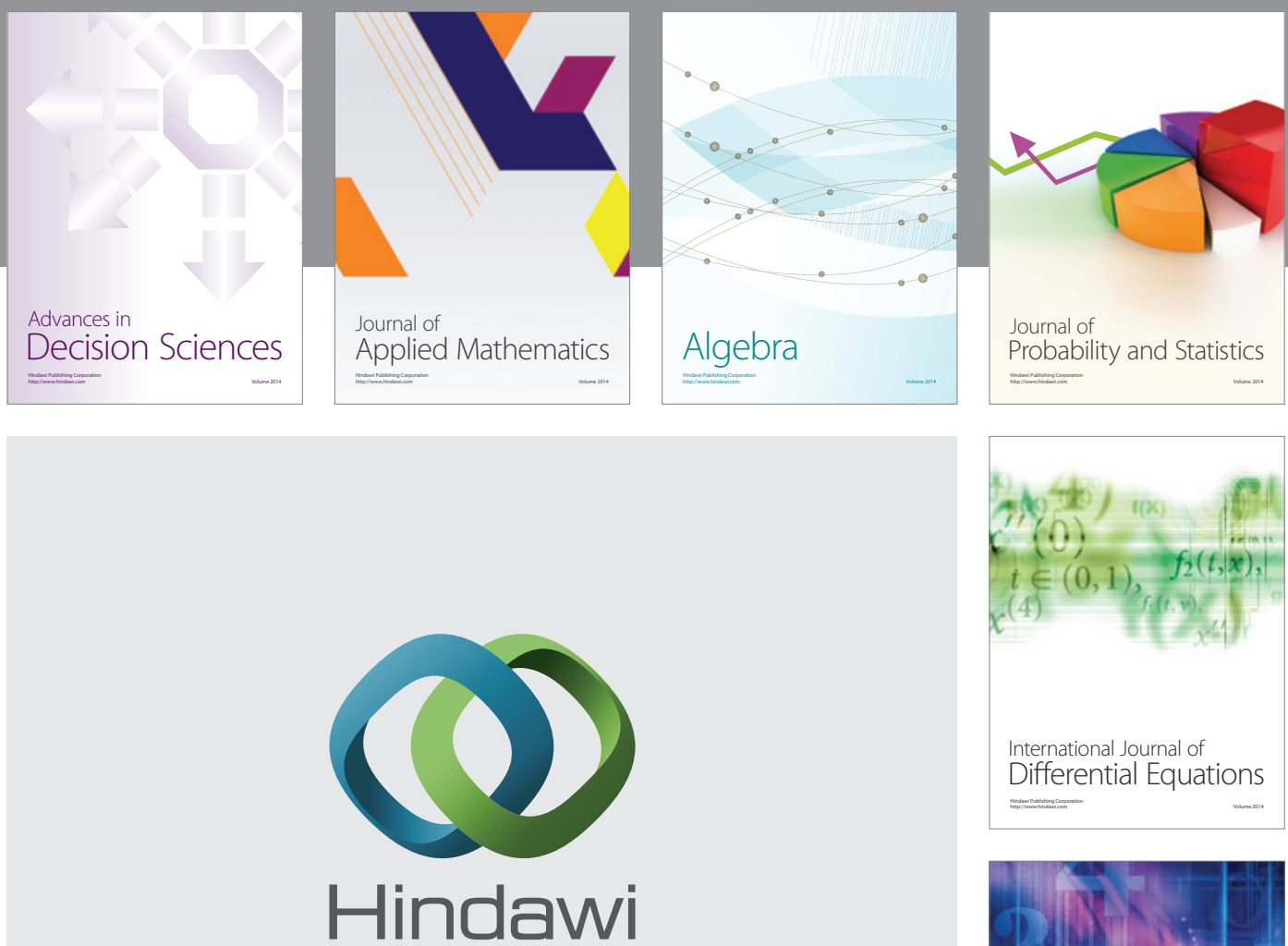

Submit your manuscripts at http://www.hindawi.com
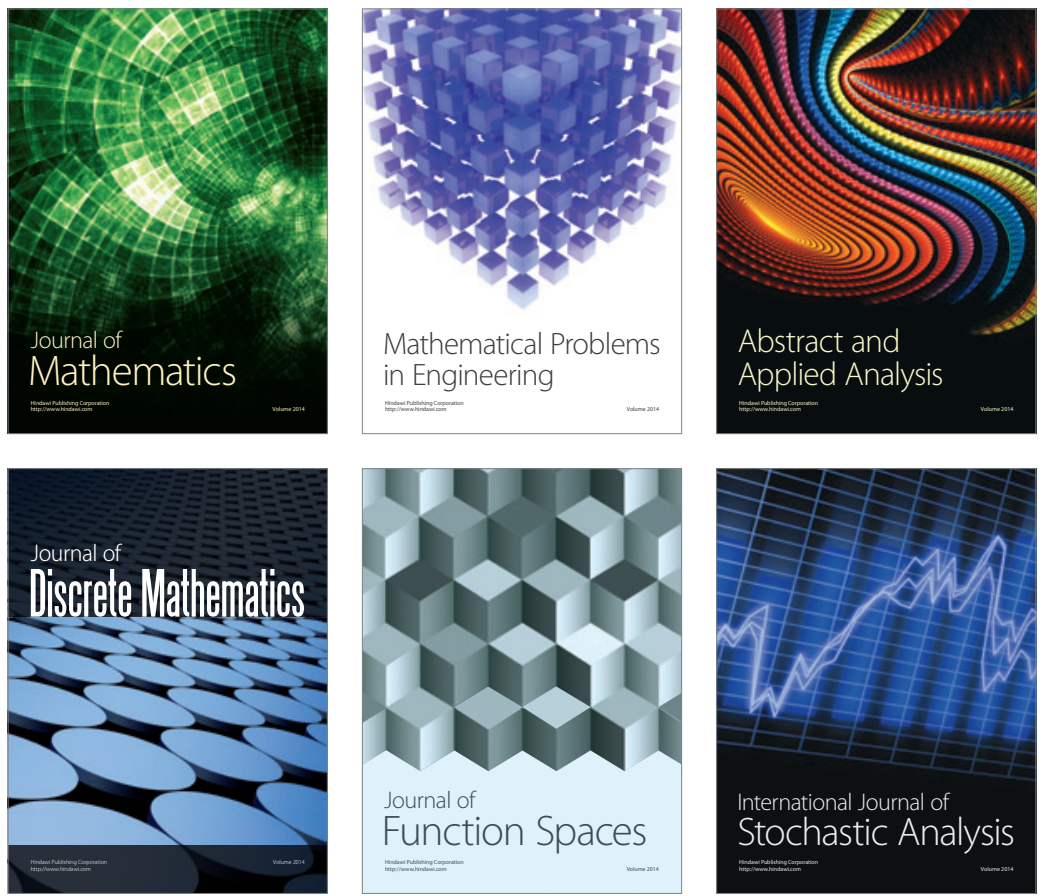

Journal of

Function Spaces

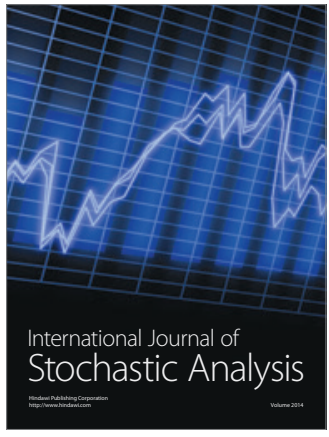

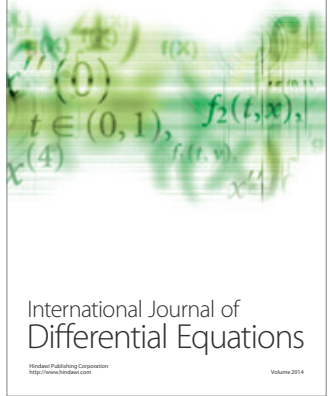
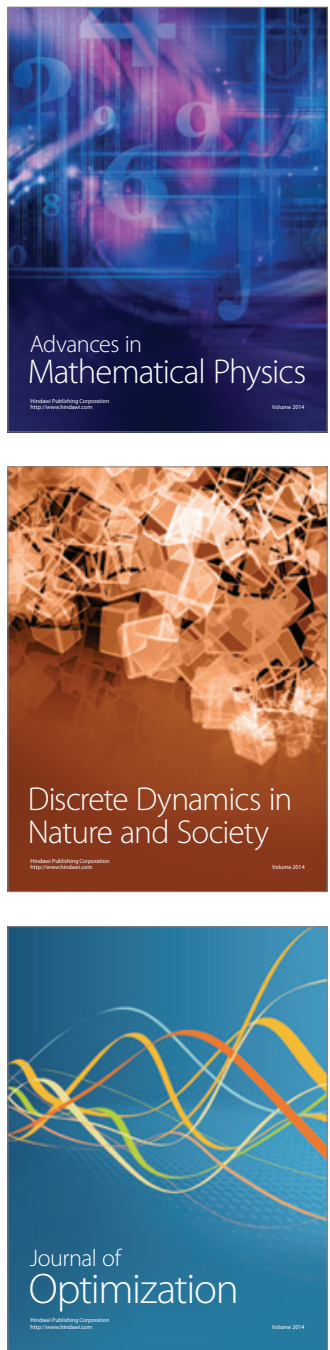